\title{
Bounded Analysis and Practical Tracking Control of Complex Stochastic Nonlinear Systems with Unknown Control Coefficients
}

\author{
Long-Chuan Guo ${ }^{1}{ }^{1}$ and Xiang-Kun Fang ${ }^{2}$ \\ ${ }^{1}$ School of Mechanical Engineering, Hangzhou Dianzi University, Hangzhou 310018, China \\ ${ }^{2}$ Beijing Shougang International Engineering Technology Co. Ltd., Beijing 100043, China \\ Correspondence should be addressed to Long-Chuan Guo; glc1988@126.com
}

Received 29 October 2019; Revised 6 January 2020; Accepted 20 January 2020; Published 13 February 2020

Academic Editor: Mohamed Boutayeb

Copyright ( $\odot 2020$ Long-Chuan Guo and Xiang-Kun Fang. This is an open access article distributed under the Creative Commons Attribution License, which permits unrestricted use, distribution, and reproduction in any medium, provided the original work is properly cited.

\begin{abstract}
This paper mainly focuses on the output practical tracking controller design for a class of complex stochastic nonlinear systems with unknown control coefficients. In the existing research results, most of the complex systems are controlled in a certain direction, which leads to the disconnection between theoretical results and practical applications. The authors introduce unknown control coefficients, and the values of the upper and lower bounds of the control coefficients are generalized by constants to allow arbitrary values to be arbitrarily large or arbitrarily small. In the control design program, the design problem of the controller is transformed into a parameter construction problem by introducing appropriate coordinate transformation. Moreover, we construct an output feedback practical tracking controller based on the dynamic and static phase combined by Ito stochastic differential theory and selection of appropriate design parameters, ensuring that the system tracking error can be made arbitrarily small after some large enough time. Finally, a simulation example is provided to illustrate the efficiency of the theoretical results.
\end{abstract}

\section{Introduction}

In the field of control theory, there are two important research directions, one is the analysis of the stability of the complex system and the other is the tracking problem of the target signal. Stability analysis is the most basic dynamic performance of a complex system, and it is also the basis for subsequent integration and analysis of the system. In actual production engineering, the complex systems, whether they are engineering systems or socioeconomic systems should be studied, mostly exhibit nonlinear characteristics. If we need to reflect the control strategy and control performance of the real system, we must consider the nonlinear characteristics of the system, which is the essential factor that affects the system control strategy and control performance. Therefore, control analysis and synthesis for nonlinear systems is an important part of the discipline of control theory and has produced quite a lot of results in recent years [1-3].
In [4], it is proposed that for the output feedback design process of nonlinear systems, whether it is stability design or tracking control design, it is necessary to restrict the growth conditions of the unmeasurable state variables in the nonlinear term. Otherwise, there is no discussion of control issues. The nonlinear growth condition is a hypothetical limitation on the nonlinear term, which is within a certain constraint. For example, there is a constant $C$ that makes the nonlinear term $f(x)$ satisfy as follows:

$$
|f(x)| \leq C\left(\left|x_{1}\right|+\left|x_{2}\right|+\cdots+\left|x_{n}\right|\right)
$$

where $x=\left(x_{1}, x_{2}, \ldots, x_{n}\right)^{T}$ are the states. Equation (1) is a typical linear growth condition. When the state variable is finitely changed, the state variable has a finite increase as a nonlinear function of the independent variable and the rate of growth satisfies certain constraints. As the most basic linear growth condition, this growth condition has been used by quite a lot of research results as the basic assumption 
for the controller design $[5,6]$. For later researchers, there is a very challenging task for nonlinear term constraints, which further weaken the constraints on nonlinear terms. The latest research hotspots focus on how to design controllers in cases where nonlinear terms satisfy the power-growth condition. In the early stage, for a nonlinear system that satisfies certain growth conditions, the state variables of the system itself are mostly measurable or partially measurable. However, in the actual system, the "black box" situation of the system is ubiquitous. In the case that the input and output are measurable and the state variables are unmeasurable, the design of the system has a very practical value. In $[5,6]$, the state feedback and output feedback control problems of nonlinear systems with unmeasurable states are studied, and the constraints of nonlinear terms are gradually relaxed.

In [7], Deng and Krstic give the design results of an output feedback controller for a class of complex stochastic nonlinear systems in which the states of the nonlinear system are measurable. Based on [7], later researchers have done a lot of research in the direction of weakening growth conditions. In [8-10], it is assumed that the nonlinear term satisfies the power-growth condition. Under this condition, the output feedback stabilization problem is studied for the complex stochastic nonlinear system, or the output tracking problem is studied. However, the above results impose many restrictions on the power term, for example, a power term must satisfy an odd condition or a nonlinear term does not depend on all system states. Krstic and Long and Zhao $[11,12]$ weakened the constraints of nonlinear terms into polynomial function growth conditions:

$$
|f(x)| \leq\left(1+\left|x_{1}\right|^{p}\right)\left(\eta_{1}\left(\left|x_{1}\right|+\cdots+\left|x_{i}\right|\right)+\eta_{2}\right),
$$

where $x_{1}$ is the output which is the only measured state. Krstic [11] studied the output feedback tracking problem of the known constant $\eta_{1}>0$ and $\eta_{2}>0$. In [12], the adaptive output feedback tracking problem for uncertain nonlinear systems is further studied. An adaptive output feedback tracking controller with an unknown growth rate is constructed by using the power integration method and the inverse push method. In the current research results, only the output feedback problem of nonlinear systems is studied. However, the deepening of the system to stochastic nonlinear systems, even stochastic nonlinear systems affected by time delays, has not been studied for the output feedback control problems of such stochastic nonlinear time-delay systems, especially how to further relax the nonlinearity. There are still many places to explore in this respect, and this paper will focus on doing some work.

Output tracking control is an important topic in control theory. It has received extensive attention in the recent decades [13-15]. Under some strong conditions imposed on the system and the tracked signal, the asymptotic stabilization implies the asymptotic tracking problem. However, for some practical systems, it is inevitable that some serious uncertainties such as unknown control directions and less reference signal information will be encountered, making it difficult or even impossible to achieve asymptotic tracking [16-19]. In this case, the practical tracking is proposed.
Unlike the asymptotic tracking, the practical tracking only requires the tracking error to be driven to the arbitrarily set small neighborhood of the origin instead of converge to zero, so practical tracking is easier to implement and it is enough for practical applications [17]. As we all know, in the existing research results, the control direction is known for the tracking control problem of nonlinear systems [18]. In [19], in the case where the reference signal and its derivative requirements are known and bounded, the author proposes some representative control schemes for the output feedback asymptotic tracking. When the reference signal information is insufficient [20,21], respectively, study the practical tracking problem of the nonlinear system. Zhang et al [22] studied a class of nonlinear systems with output coefficients of unknown coefficients. Zhang et al [23] considered a class of generalized strict feedback uncertain systems. However, in the practical system, the situation in the unknown control direction is ubiquitous, such as attitude control of the spacecraft, military radar tracking control, precision guidance control, and industrial robot control [24-28]. In theoretical research, the unknown control direction can be defined by the upper and lower bounds. Under the different conditions of the reference signal and the control coefficient, Liang et al [26] considered the practical tracking problem of high-order nonlinear systems. It should be pointed out that the direction of the unknown control coefficients in [26] has a known control direction.

Most research results are only limited to the nonlinear systems. Naturally, one may ask an interesting and challenging problem: If we consider the tracking control problem for stochastic nonlinear system, how to design a controller? As is well known, the stochastic factor is a main resource that contributes significantly to system complexity. In some practical fields, such as aerospace, biological, economic systems, and health community, the output tracking problem for these stochastic nonlinear systems has a wide range of practical applications.

Based on the theory of stochastic nonlinear systems, this paper mainly studies the output feedback tracking control problem of a class of stochastic nonlinear systems with unknown coefficients. Here, we assume that the nonlinear term satisfies the growth condition of the output polynomial function and constructs the output based on the combination of dynamic and static. The feedback tracking controller ensures that the system tracking error converges to a small neighborhood of zero. It is important to note that the system has an unknown direction of control. Due to the existence of linear uncontrollable modalities in this nonlinear system, the practical output tracking of nonlinear systems with unknown control directions is still unsolved and has practical industrial application significance.

The main innovative contributions of this paper are as follows:

(1) In the previous research results, most of the systems studied are for the system determined by the control coefficient. Even for uncertain systems, the control function has the control direction and control coefficient [29]. Guo [29] focused on the output 
feedback practical tracking problem for a class of stochastic nonlinear systems in which the coefficient in front of the control action $u$ is 1 . Such system's design greatly limits the scope of design and application of the system controller, especially in the actual system. Because in many practical systems, such as aerospace systems, terrestrial satellite navigation systems, industrial processes, and other complex systems, the control role mostly contains uncertain factors. The uncertainty here does not only refer to the uncertainty of the system itself, but to the uncertainty of the system control. Therefore, it is necessary to study the uncertainty of the control effect. Based on this consideration, we consider the output feedback practical tracking problem for a class of stochastic nonlinear systems with unknown control directions. The system studied in this paper introduces unknown control coefficients, and the values of the upper and lower bounds of the control coefficients are generalized by constants to allow arbitrary values to be arbitrarily large or arbitrarily small. By introducing a new high-gain adjustment law, the design method of the global adaptive actual tracking control for such uncertain nonlinear systems is given.

(2) In the control design program of this paper, the design problem of the controller is transformed into a parameter construction problem by introducing appropriate coordinate transformation. We do not use an iterative-based prepush and saturation control design method because the controller gain obtained by the iterative design method may become too small during multiple iterations, and the resulting controller is theoretically effective, but it is difficult to achieve in engineering practice and when the system's dimension is relatively high, multiple iterations will greatly increase the design difficulty and complexity. The controller designed in this paper is simple in form and moderate in dynamic/ static gain, so it is easier to apply to engineering practice.

(3) All the mentioned references are only limited to the nonlinear systems $[30,31]$. As is well known, the stochastic factor is a main resource that contributes significantly to system complexity. Based on the research of the output tracking control problem for nonlinear systems, the concept of introducing random factors is proposed to study the output tracking actual tracking control problem of a class of stochastic nonlinear systems and the original harsh growth conditions are removed. It satisfies the more general polynomial function growth condition. Under this condition, the static and dynamic output feedback actual tracking controller design scheme is proposed to make the output tracking error converge into the small neighborhood of zero.
This paper is organized as follows: Section 2 gives the description of the stochastic nonlinear system and control objectives. Section 3 gives the design process and results of the output feedback practical tracking controller, which mainly includes state observer design and boundedness analysis of system states and observer gain. Section 4 gives a simulation example to verify the effectiveness of the output feedback tracking controller. Section 5 summarizes this paper and prospects for future work.

\section{Problem Description}

This paper considers the output feedback practical tracking problem for a class of stochastic nonlinear systems with unknown control directions as follows:

$$
\begin{aligned}
\mathrm{d} x_{i} & =g_{i}(t, x) x_{i+1} \mathrm{~d} t+f_{i}(t, x, u) \mathrm{d} \omega, \quad(i=1, \ldots, n-1), \\
\mathrm{d} x_{n} & =g_{n}(t, x) u \mathrm{~d} t+f_{n}(t, u, x) \mathrm{d} \omega, \\
y & =x_{1}-y_{r}(t),
\end{aligned}
$$

where $x=\left(x_{1}, \ldots, x_{n}\right)^{T} \in R^{n}, u \in R, y \in R$ are the states, input, and output of the system; corresponding to this, $y_{r}$ is a given unmeasurable output trajectory; here, we introduce a stochastic process for the system: $\omega$ is an $m$ dimensional standard Wiener process defined on the complete probability space $(\Omega, \Gamma, \mathrm{P})$ with $\Omega$ being a sample space, $\Gamma$ being a filtration, and $\mathrm{P}$ being a probability measure; $g_{i}(t, x),(i=1, \ldots, n)$ is the control coefficient and is bounded; furthermore, the control coefficient is unknown and the first variable is continuous and the second variable is locally Lipschitz; nonlinear term $f_{i}: R^{+} \times R \times R^{n} \longrightarrow R^{m}, i=1, \ldots, n$ is continuous for $t$ and locally Lipschitz in $(u, x)$.

The main goal of this paper is to design an output controller so that the stochastic nonlinear system (3) tracking error can be made arbitrarily small after some large enough time. In order to achieve the control objectives, the system and reference signals are required to satisfy the following assumptions.

Assumption 1. There exists a positive integer $p$ and a known positive constant $c_{0}$ such that the following inequality holds:

$$
\begin{array}{r}
\left|f_{i}(t, x, u)\right| \leq c_{0}\left(1+\left|x_{1}\right|^{p}\right)\left(\left|x_{1}\right|+\cdots+\left|x_{i}\right|\right)+c_{0}, \\
i=1, \ldots, n .
\end{array}
$$

Based on this assumption, system (3) is dominated by an output polynomial function growth rate system.

Assumption 2. The symbol of $g_{i}(t, x),(i=1, \ldots, n)$ is defined as the control direction of system (3), and control direction in this system is unknown. $g_{i}(t, x)$ takes the value on the unknown interval $\left[\xi_{i}, \zeta_{i}\right]$ in which $\xi_{i}$ and $\zeta_{i}$ are the upper and lower bounds of the determined constant interval. 
Assumption 3. The reference output trajectory $y_{r}$ of system (3) is continuously differentiable and satisfies the following inequality:

$$
\max \left(\left|y_{r}\right|,\left|\dot{y}_{r}\right|\right) \leq c_{1},
$$

where $c_{1}$ is known as the positive constant.

According to the characteristics and constraints of the above system, it leads to the objective of this paper: for any constant $\delta>0$, all the states of the stochastic nonlinear system (3) are well defined and bounded. In addition, there exists a finite time $T>0$ such that for any $t>T$, we get

$$
|y(t)|=\left|x_{1}(t)-y_{r}(t)\right| \leq \delta \text {. }
$$

Remark 1. System (3) studied in this paper is a stochastic nonlinear system after introducing stochastic factors. System (3) is observable according to assumption 1 which is dependent on the unmeasurable state, and according to assumption 3, it can be seen that for the reference trajectory, only the upper bound value and the upper bound value of the derivative are given, which means that it is not necessary to give a specific description function of the reference trajectory or give more information. Due to the insufficiency of the system and tracking signal information and the system instability caused by the introduction of stochastic factors, the general tracking control method, such as the asymptotic tracking control method, can no longer solve the problems in this section. For the abovechallenging problems, we use the static and dynamic gain control design method to study the problem and introduce the appropriate coordinate transformation to transform the design problem of the controller into a parameter construction problem.

\section{Adaptive Practical Tracking Control Design}

According to the description of the stochastic nonlinear system with unknown control coefficients (1) and Assumptions $1-3$, we get

$$
\left|f_{i}(t, x, u)\right| \leq c\left(1+|y|^{p}\right)\left(\left|x_{1}\right|+\cdots+\left|x_{i}\right|\right)+c
$$

where $c=c_{0} \max \left(1+2^{p-1} c_{1}^{p}, 2^{p-1}\right)$ is a known constant.

For the sake of convenience of calculation and formula derivation, the following simple state transformation is introduced, and then it can obtain updated stochastic nonlinear system:

$$
\begin{aligned}
\mathrm{d} z_{i} & =g_{i}(t, z) z_{i+1} \mathrm{~d} t+F_{i}(t, u, z) \mathrm{d} \omega, \\
\mathrm{d} z_{n} & =g_{n}(t, z) u \mathrm{~d} t+F_{n}(t, u, z) \mathrm{d} \omega, \\
y & =z_{1} .
\end{aligned}
$$

Based on the simplicity of formula derivation, especially introducing state transitions:

$$
\begin{aligned}
& z_{1}=x_{1}-y_{r} \\
& z_{i}=x_{i}, \quad i=2, \ldots, n,
\end{aligned}
$$

definition $F_{i}(t, u, z)$ is as follows:

$$
F_{i}(t, u, z)=g_{i} f_{i}\left(t, u, z_{1}+y_{r}, z_{2}, \ldots, z_{n}\right)
$$

States observer of system (3) is established as

$$
\begin{aligned}
& \dot{\hat{z}}_{i}=\widehat{z}_{i+1}+K^{i} l_{i}\left(z_{1}-\widehat{z}_{1}\right), \quad i=1, \ldots, n-1, \\
& \dot{\vec{z}}_{n}=u+K^{n} l_{n}\left(z_{1}-\widehat{z}_{1}\right),
\end{aligned}
$$

where $l_{i}>0(i=1, \ldots, n)$ is the coefficient of the Hurwitz polynomial with $s^{n}+l_{1} s^{n-1}+\cdots+l_{n-1} s+l_{n} . K=R T(t)$ is a high-order gain term which consists of a constant $R$ and a variable $T(t)$ as follows:

$$
\dot{T}=-\alpha_{1} T^{2}+\alpha_{2}\left(1+|y|^{p}\right)^{2} T, \quad T(0)=1 .
$$

Define the system error states as $e_{i}=z_{i}-\widehat{z}_{i}(i=$ $1, \ldots, n)$, and then by (7)-(10), the error system can be obtained as follows:

$$
\begin{aligned}
\mathrm{d} e_{i} & =\|\xi\| \cdot\|\zeta\| e_{i+1} \mathrm{~d} t-K^{i} l_{i} e_{1} \mathrm{~d} t+F_{i}(t, u, z) \mathrm{d} \omega \\
\mathrm{d} e_{n} & =-\|\xi\| \cdot\|\zeta\| K^{n} l_{n} e_{1} \mathrm{~d} t+F_{n}(t, u, z) \mathrm{d} \omega .
\end{aligned}
$$

Remark 2. The uncertainty of the control coefficient is a major theoretical problem that plagues the industry. Based on the past research results [25], this paper designs a control coefficient with certainty $g$. In [25], the output feedback tracking control of such uncertain nonlinear systems is studied. The author introduces coordinate transformation, obtains a continuous adaptive controller by means of the Nussbaum gain method and increased power integration method, and finally guarantees the system tracking error. After a finite time, it converges to the small neighborhood of zero, and at the same time makes the state of the closed-loop nonlinear system satisfy the boundedness. Drawing on the above research results, we use the deterministic interval segmentation theory to effectively constrain the unknown control coefficients.

Transformation of estimated states $\widehat{z}_{i}$ and error states $e_{i}$ is introduced to further simplify subsequent controller design issues:

$$
\begin{gathered}
\varepsilon_{i}=\frac{e_{i}}{K^{b+i-1}}, \quad i=1, \ldots, n, \\
\tau_{i}=\frac{\widehat{z}_{i}}{K^{b+i-1}}, \quad i=1, \ldots, n,
\end{gathered}
$$

where $0 \leq b \leq(1 / p)\|\xi\| \zeta\|\|$ is known as the constant. Further, by (13), systems (8)-(10) can be converted into

$$
\begin{aligned}
\mathrm{d} \varepsilon & =K L\|\xi\|\|\zeta\| \varepsilon \mathrm{d} t-\frac{\dot{K}}{K} C_{b} \varepsilon \mathrm{d} t+G(z, K) \mathrm{d} \omega, \\
\dot{\tau} & =K L^{T} \tau\|\xi\|\|\zeta\|+K l \varepsilon_{1}-\frac{\dot{K}}{K} C_{b} \tau,
\end{aligned}
$$

where 


$$
\begin{aligned}
L & =\left(\begin{array}{cccc}
-l_{1} & 1 & \cdots & 0 \\
\vdots & \vdots & \ddots & \vdots \\
-l_{n-1} & 0 & \cdots & 1 \\
-l_{n} & 0 & \cdots & 0
\end{array}\right), \\
L^{T} & =\left(\begin{array}{cccc}
0 & 1 & \cdots & 0 \\
\vdots & \vdots & \ddots & \vdots \\
0 & 0 & \cdots & 1 \\
-l_{1} & -l_{2} & \cdots & -l_{n}
\end{array}\right), \\
C_{b} & =\operatorname{diag}(b, b+1, \ldots, b+n-1), \\
G(z, K) & =\left(\frac{F_{1}}{K^{b}}, \ldots, \frac{F_{n}}{K^{b+n-1}}\right)^{T} .
\end{aligned}
$$

According to the system description and definition in the second section, it is concluded that the closed-loop system has a unique solution in interval $\left[0, t_{T}\right)$. Moreover, if $T$ is a maximum value of $t_{f}$, then $0<T \leq \infty$.

Next, we need to discuss the boundedness of the various states and parameter variables of the system. Appropriate parameters $r_{1}, r_{2}, r_{3}$, and $r_{4}$ are chosen such that the positive definite matrix $P, Q$ and matrix $L, L^{T}, C_{b}$ satisfy the following relation:

$$
\begin{aligned}
& r_{1} I_{n} \leq C_{b} P+P C_{b} \leq r_{2} I_{n}, \\
& r_{3} I_{n} \leq C_{b} Q+Q C_{b} \leq r_{4} I_{n} .
\end{aligned}
$$

Define the following Ito Lyapunov function $V(\varepsilon, \tau)=$ $\varepsilon^{T} P \varepsilon+\tau^{T} Q \tau$, and then it can get the trajectory of (17) along the Ito differentiation for system (14) as follows:

$$
\begin{aligned}
L V= & -K|\varepsilon|^{2}\|\zeta\|\|\xi\|+2 \varepsilon^{T} P G-\frac{\dot{K}}{K} \varepsilon^{T}\left(C_{b} P+P C_{b}\right) \varepsilon-2 K|\tau|^{2} \\
& \cdot\|\zeta\|\|\xi\|+2 K \tau^{T} \varepsilon_{1} h-\|\zeta\|\|\xi\| \frac{\dot{K}}{K} \tau^{T}\left(C_{b} Q+Q C_{b}\right) \tau \\
& -\frac{\dot{K}}{K} \varepsilon^{T}\left(C_{b} P+P C_{b}\right) \varepsilon \\
\leq & r_{4} \alpha_{1} B\|\zeta\|\|\xi\||\tau|^{2}-r_{3} \alpha_{2}\left(1+|y|^{p}\right)^{2}|\tau|^{2},
\end{aligned}
$$

where

$$
|G|=\left(\left(\frac{F_{1}}{K_{b}}\right)^{2}+\cdots+\left(\frac{F_{n}}{K^{b+n-1}}\right)^{2}\right)^{1 / 2},
$$

where (18) can be magnified to

$$
\begin{aligned}
& (\|P\|+\|G\|)\left(c\left(1+|y|^{p}\right)\left(\sqrt{n}(|\varepsilon|+|\tau|)+c_{1}\right)+c+c_{1}\right)^{2} \\
& \leq(\|P\|+\|G\|) \cdot\left(n c^{2}\left(1+|y|^{p}\right)^{2}\left(|\varepsilon|^{2}+|\tau|^{2}\right)\right. \\
& \left.\quad+4 c^{2}\|\zeta\|\|\xi\| c_{1}^{2}+2\left(c+c_{1}\right)^{2}\right) .
\end{aligned}
$$

By the inequality scaling principle, parameter $\alpha_{2}$ can be chosen as follows:

$$
\alpha_{2} \geq \frac{\|\zeta\|\|\xi\| c\|P\|\left(1+c_{1}\right)-n c^{2}(\|P\|+\|Q\|)}{r_{3}} .
$$

Since $T \geq 1$, parameter $R$ needs to satisfy the following relationship:

$$
R \geq \frac{\|\zeta\|\|\xi\|\|\| P \|\left(n c+c+c_{1}\right)+r_{2} \alpha_{1}}{1-\|\zeta\|\|\xi\||l|^{2}} \geq 1 .
$$

According to the above choice of parameters, (22) can be translated into

$$
\begin{aligned}
L V \leq & -\frac{R}{\max \left(\lambda_{\max }(P)+\lambda_{\max }(Q)\right)} \cdot V \\
& +\|\zeta\|\|\xi\|\|P\|\left(c c_{1}+c+c_{1}\right)+\left(c^{2}\left(1+|y|^{p}\|\zeta\|\|\xi\|\right)^{2} c_{1}^{2}\right. \\
& \left.+\left(c+c_{1}\right)^{2}\right)(\|P\|+\|Q\|) .
\end{aligned}
$$

By using other variables instead of the above derivation results, we get

$$
\begin{aligned}
& E_{1}=\frac{R}{\max \left(\lambda_{\max }(P)+\lambda_{\max }(Q)\right)}, \\
& E_{2}=\|\zeta\|\|\xi\|\|P\|\left(c c_{1}+c+c_{1}\right)+\left(\left(1+|y|^{p}\right)^{2} c_{1}^{2}+1\right) .
\end{aligned}
$$

Since the closed-loop system has a unique solution in interval $[0, \infty)$, by $(22)-(24)$, it gives

$$
V(\varepsilon(t), \tau(t)) \leq V(\varepsilon(0), \tau(0)) e^{-\left(E_{1} / A\right) t}+\frac{E_{2}}{E_{1}}, \quad t \in[0, T),
$$

such that states $\varepsilon(t)$ and $\tau(t)$ of the stochastic nonlinear system (8)-(10) and (14) are well defined and bounded on $[0, T)$.

Correspondingly, by $y=z_{1}=\left(\varepsilon_{1}+\tau_{1}\right) K^{b}$ and $|y| \leq$ $(R T)^{b}\left(\left|\varepsilon_{1}\right|+\left|\tau_{1}\right|\right)$, it can get $|y| \leq(A B)^{b} E_{3}$. According to the boundedness of $\varepsilon$ and $\tau$ on $[0, T)$, combined with the above obtained $|y| \leq(R T)^{b} E_{3}$ and $b \in(0,(1 / p)\|\zeta\|\|\xi\|)$, the following is obtained:

$$
T(t) \leq \frac{\alpha_{2}^{2}\|\zeta\|\|\xi\|\left(1+E_{3}^{2 p} \sqrt{R}\right)^{2}}{\alpha_{1}^{2}} .
$$

Through the above analysis, it can prove that the system gain $T(t)$ is well defined and bounded on $[0, T)$ with $T=\infty$.

Through the above analysis, it can be concluded that the system is essentially bounded. Then, we give the theorem of output feedback practical tracking controller.

Theorem 1. Consider a class of the stochastic nonlinear system (3) with unknown control coefficients under Assumption 1-3. By choosing appropriate parameters $a_{i}, l_{i}, \alpha_{1}$, and $\alpha_{2}$, the following output feedback practical tracking controller can be designed as follows: 


$$
u=-\left(K^{n} a_{1} \widehat{z}_{1}+K^{n-1} a_{2} \widehat{z}_{2}+\cdots+K a_{n} \widehat{z}_{n}\right),
$$

in which $K$ is defined by (11) and $a_{i}>0$ is the coefficient of Hurwitz polynomial. For any $\delta>0$, there exists a finite time $T$ such that $y=\left|x_{1}-y_{r}\right| \leq \delta$ and all the states of stochastic nonlinear system (8)-(10) and (14) are bounded on $[0, T)$ by an output feedback practical tracking controller (27).

Proof. Since the closed-loop stochastic nonlinear system satisfies the locally Lipschitz condition, according to [32], it is concluded that the closed-loop system has a unique solution in interval $\left[0, t_{T}\right)$. Moreover, if $T$ is a maximum value of $t_{f}$, then $0<T \leq \infty$.

There exists a time $T=T_{1}$ such that

$$
V(\varepsilon(t), \tau(t)) \leq \frac{2 E_{2}}{E_{1}}, \quad t \geq T=T_{1} .
$$

According to (20)-(26), it can conclude that

$$
\lambda_{\min }(P)|\varepsilon|^{2}+\lambda_{\min }(Q)|\tau|^{2} \leq V(\varepsilon(t), \tau(t)) .
$$

Further, squaring the above formula (29), we get

$$
\varepsilon_{1}^{2}(t)+\tau_{1}^{2}(t) \leq \frac{E_{4}}{R} .
$$

By using $|y| \leq(A B)^{b} E_{3}$ and (28)-(30), we get

$$
|y| \leq A^{b} B^{b}\|\xi\| \zeta\|\|\left(\left|\varepsilon_{1}(t)\right|+\left|\tau_{1}(t)\right|\right) \leq \sqrt{\frac{\|\xi\| \zeta\|\| E_{4}}{A}} .
$$

Simultaneously, combined with (31), we obtain

$$
y^{2}(t) \leq \frac{\|\xi\| \zeta\|\| E_{4}}{A} .
$$

Through the above proof and analysis, the output $|y(t)|$ of the stochastic nonlinear system (3) can be gradually converged to zero by choosing an appropriate gain $R$ and $T$.

Proof. Theorem 1. has been completed such that all the system states are bounded, and the tracking error can be made arbitrarily small after some large enough time.

\section{Simulation Example}

To verify the output feedback tracking controller designed in Section 3, this section considers a class of 2-dimensional stochastic nonlinear systems as given as follows:

$$
\begin{aligned}
\mathrm{d} x_{1} & =\sin x_{1} x_{2} \mathrm{~d} t+\mathrm{d} \omega, \\
\mathrm{d} x_{2} & =\sin \left(x_{1}+x_{2}\right) u \mathrm{~d} t+x_{2} \ln \left(1+0.5 x_{1}^{2}\right) \mathrm{d} \omega, \\
y & =x_{1}-y_{r},
\end{aligned}
$$

where the control coefficient $g_{1}(x, t)=\sin x_{1}, g_{2}(x, t)=$ $\sin \left(x_{1}+x_{2}\right), c_{0}=c_{1}=0.5 p=2$. This shows that $g_{1}(x, t)$ and $g_{2}(x, t)$ belong to $[-1,1]$, and $\xi=\zeta=1$. This simulation process sets the target tracking path of the system to $y_{r}(t)=\sin t$.

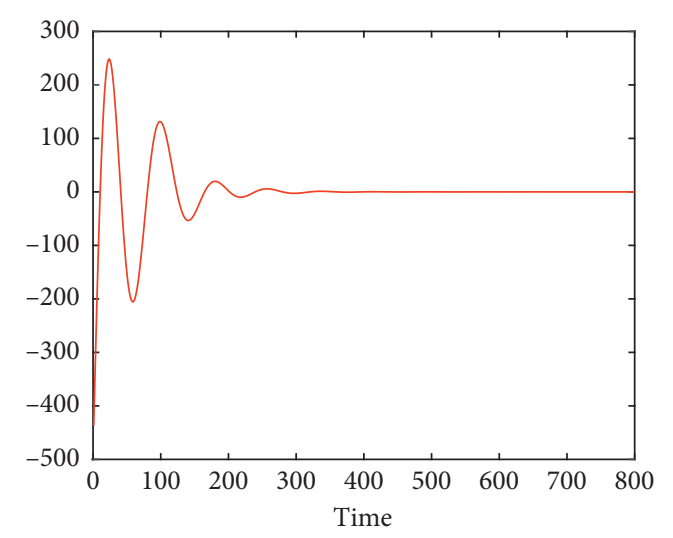

FIGURE 1: The trajectory of system output $y=x_{1}-y_{r}$.

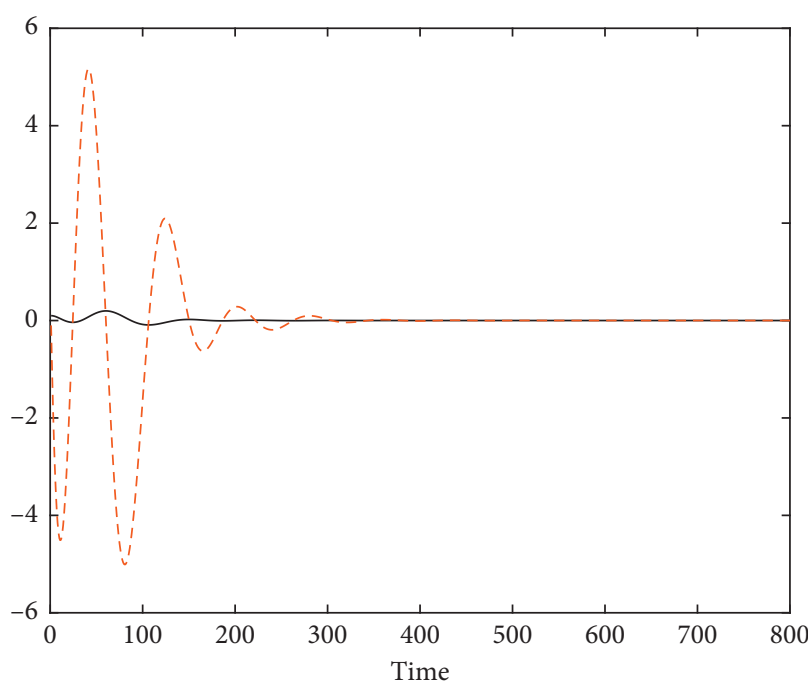

$\begin{array}{ll}- & x_{1} \\ --- & x_{2}\end{array}$

FIGURE 2: The trajectory of system state $x_{1}$ and $x_{2}$.

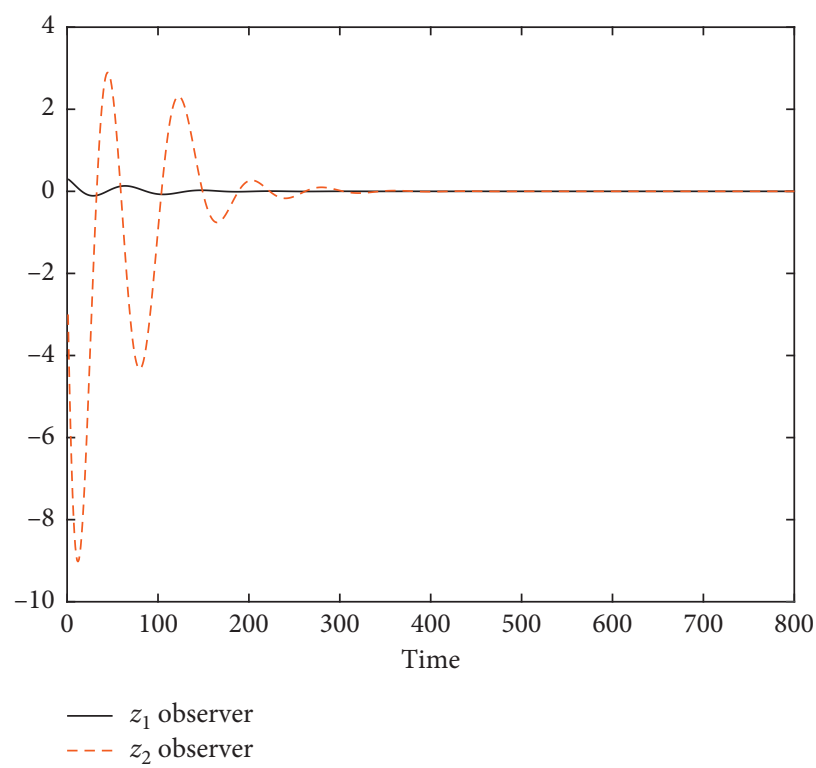

FIGURE 3: The trajectory of system observer state $\widehat{z}_{1}$ and $\widehat{z}_{2}$. 
Further, the parameters of the output feedback tracking controller are set according to Theorem 1 . Then, start the system simulation and select the initial value:

$$
\left[x_{1}(0), x_{2}(0), \widehat{z}_{1}(0), \widehat{z}_{2}(0)\right]=[0.1,-0.3,0.3,-3] \text {. }
$$

Figures 1-3show the response curve of the closed-loop system 1 . Observed by the simulation diagram, the state of the two-dimensional system () is bounded and gradually converges, and the system can also be observed. The output tracking error gradually converges and finally adjusts to near zero. The simulation results verify the effectiveness of the designed output feedback actual tracking controller.

\section{Concluding and Future Prospects}

This paper mainly focuses on the output practical tracking controller design for a class of complex stochastic nonlinear systems with the unknown control coefficient. It is worth noting that this paper expands the uncertainty of the system, making the research results more effective in the actual system. We introduce unknown control coefficients, and the values of the upper and lower bounds of the control coefficients are generalized by constants to allow arbitrary values to be arbitrarily large or arbitrarily small. In the control design program of this paper, the design problem of the controller is transformed into a parameter construction problem by introducing appropriate coordinate transformation. Moreover, we extend it to stochastic nonlinear systems and construct an output feedback practical tracking controller based on the dynamic and static phase combined by Ito stochastic differential theory and selection of appropriate design parameters, ensuring that the system tracking error can be made arbitrarily small after some large enough time. Finally, a simulation example is provided to illustrate the efficiency of the theoretical results.

In the future, we will expand our horizons into other fields such as chemical process systems and power electronics systems and construct a conforming actual system through mechanism modeling. At the same time, it is aimed at the instability of the financial system, the fluctuation of the price system, such as the phenomenon of time lag in the stock system, etc., to explore whether the results of this paper can be applied to these practical examples.

\section{Data Availability}

All data generated or analyzed during this research are included in this paper.

\section{Conflicts of Interest}

The authors declare that there are no conflicts of interest regarding the publication of this paper.

\section{Acknowledgments}

This work was supported in part by the National Natural Science Foundation of China, under Grant 61807010,
Natural Science Foundation of Zhejiang Province of China (Grant LQ18F030007), and Zhejiang Province Postdoctoral Funding Project (Grant ZJ20180026).

\section{References}

[1] Y. Mizuta and T. Shimomura, "Sobolev's inequality for riesz potentials with variable exponent satisfying a log-hölder condition at infinity," Journal of Mathematical Analysis and Applications, vol. 311, no. 1, pp. 268-288, 2005.

[2] J.-Q. Li, Y.-S. Zhang, C.-M. Ji, A.-J. Wang, and J. R. Lund, "Large-scale hydropower system optimization using dynamic programming and object-oriented programming: the case of the northeast China power grid," Water Science and Technology, vol. 68, no. 11, pp. 2458-2467, 2013.

[3] Q. Liu and Z. Liang, "Global stabilisation of a class of feedforward systems with distributed delays," IET Control Theory \& Applications, vol. 9, no. 1, pp. 140-146, 2015.

[4] F. Mazenc, L. Praly, and W. P. Dayawansa, "Global stabilization by output feedback: examples and counterexamples," Systems \& Control Letters, vol. 23, no. 2, pp. 119-125, 1994.

[5] M. Abbaszadeh and H. J. Marquez, "LMI optimization approach to robust $H$ infinity observer design and static output feedback stabilization for discrete-time nonlinear uncertain systems," International Journal of Robust and Nonlinear Control, vol. 19, no. 3, pp. 313-340, 2009.

[6] A. Benabdallah, M. A. Hammami, and J. Kallel, "Robust stability of uncertain piecewise-linear systems: LMI approach," Nonlinear Dynamics, vol. 63, no. 1-2, pp. 183-192, 2011.

[7] D. Soudry, D. Di Castro, A. Gal, A. Kolodny, and S. Kvatinsky, "Memristor-based multilayer neural networks with online gradient descent training," IEEE Transactions on Neural Networks and Learning Systems, vol. 26, no. 10, pp. 2408-2421, 2015.

[8] C. Briat, "Stability analysis and control of a class of LPV systems with piecewise constant parameters," Systems \& Control Letters, vol. 82, pp. 10-17, 2015.

[9] P. Kokotovic and M. Arcak, "Constructive nonlinear control: a historical perspective," Automatica, vol. 37, no. 5, pp. 637-662, 2001.

[10] T. Chen and J. Huang, "A small gain approach to global stabilization of nonlinear feedforward systems with input unmodeled dynamics," Automatica, vol. 46, no. 6, pp. 10281034, 2010.

[11] M. Krstic, "Feedback linearizability and explicit integrator forwarding controllers for classes of feedforward systems," IEEE Transactions on Automatic Control, vol. 49, no. 10, pp. 1668-1682, 2004.

[12] L. Long and J. Zhao, "Global stabilization for a class of switched nonlinear feedforward systems," Systems \& Control Letters, vol. 60, no. 9, pp. 734-738, 2011.

[13] X. Ye, "Adaptive stabilization of time-delay feedforward nonlinear systems," Automatica, vol. 47, no. 5, pp. 950-955, 2011.

[14] H. Ye, "Saturated delayed controls for feedforward nonlinear systems," IEEE Transactions on Automatic Control, vol. 59, no. 6, pp. 1646-1653, 2014.

[15] B. Bao, F. Hu, and Z. Liu, "Mapping equivalent approach to analysis and realization of memristor-based dynamical circuit," Chinese Physics B, vol. 23, no. 7, pp. 303-310, 2014.

[16] M.-C. Pai, "RBF-based discrete sliding mode control for robust tracking of uncertain time-delay systems with input nonlinearity," Complexity, vol. 21, no. 6, pp. 194-201, 2015. 
[17] L. Praly and Z. P. Jiang, "Linear output feedback with dynamic high gain for nonlinear systems," Systems \& Control Letters, vol. 53, no. 2, pp. 107-116, 2004.

[18] P. Krishnamurthy and F. Khorrami, "Feedforward systems with ISS appended dynamics: adaptive output-feedback stabilization and disturbance attenuation," IEEE Transactions on Automatic Control, vol. 53, no. 1, pp. 405-412, 2008.

[19] M.-S. Koo, H.-L. Choi, and J.-T. Lim, "Global regulation of a class of uncertain nonlinear systems by switching adaptive controller," IEEE Transactions on Automatic Control, vol. 55, no. 12, pp. 2822-2827, 2010.

[20] X. Zhang and Y. Lin, "Global adaptive stabilisation of feedforward systems by smooth output feedback," IET Control Theory \& Applications, vol. 6, no. 13, pp. 2134-2141, 2012.

[21] X. Zhang, Q. Liu, L. Baron, and E.-K. Boukas, "Feedback stabilization for high order feedforward nonlinear time-delay systems," Automatica, vol. 47, no. 5, pp. 962-967, 2011.

[22] X. Zhang, L. Baron, Q. Liu, and E.-K. Boukas, "Design of stabilizing controllers with a dynamic gain for feedforward nonlinear time-delay systems," IEEE Transactions on Automatic Control, vol. 56, no. 3, pp. 692-697, 2011.

[23] X. Zhang, H. Gao, and C. Zhang, "Global asymptotic stabilization of feedforward nonlinear systems with a delay in the input," International Journal of Systems Science, vol. 37, no. 3, pp. 141-148, 2006.

[24] H.-W. Jo, H.-L. Choi, and J.-T. Lim, "Observer based output feedback regulation of a class of feedforward nonlinear systems with uncertain input and state delays using adaptive gain," Systems \& Control Letters, vol. 71, pp. 44-53, 2014.

[25] W. Zha, J. Zhai, and S. Fei, "Global output feedback control for a class of high-order feedforward nonlinear systems with input delay," ISA Transactions, vol. 52, no. 4, pp. 494-500, 2013.

[26] Z. Liang and Q. Liu, "Design of stabilizing controllers of upper triangular nonlinear time-delay systems," Systems \& Control Letters, vol. 75, pp. 1-7, 2015.

[27] L.-C. Guo, X. Zuo, J.-W. Liu, and H.-Q. Liang, "Outputfeedback control of a class of stochastic nonlinear systems with power growth conditions," International Journal of Control, Automation and Systems, vol. 12, no. 2, pp. 274-282, 2014.

[28] L. Guo, J. Liu, X. Zuo, and H. Liang, "Output feedback stabilization for a special class of stochastic nonlinear time-delay system with more general growth conditions," Journal of Dynamic Systems Measurement and Control, vol. 136, no. 6, Article ID 061004, 2014.

[29] L.-C. Guo, "Practical tracking control for stochastic nonlinear systems with polynomial function growth conditions," Automatika, vol. 60, no. 4, pp. 443-450, 2019.

[30] R. Sakthivel, B. Kaviarasan, P. Selvaraj, and H. R. Karimi, "EID-based sliding mode investment policy design for fuzzy stochastic jump financial systems," Nonlinear Analysis: Hybrid Systems, vol. 31, no. 2, pp. 100-108, 2019.

[31] R. Sakthivela, H. R. Karimic, K. Raajananthini, P. Selvarajb, and Y. Ren, "Observer-based tracking control for switched stochastic systems based on a hybrid 2-D model," International Journal of Robust and Nonlinear Control, vol. 28, no. 2, pp. 478-491, 2018.

[32] R. Z. Hasminskii, Stochastic Stability of Differential Equations, Kluwer Academic Publishers, Boston, MA, USA, 1980. 